\title{
Die idiopathische Lungenfibrose
}

\author{
Idiopathic Pulmonary Fibrosis
}

\author{
A. Prasse $e^{1,2}$ \\ 1 Medizinische Hochschule Hannover, Abteilung für Pneumologie \\ 2 Fraunhofer ITEM; Clinical Research Center Hannover
}

VNR

2760512015147122481

Bibliografie

DoI http://dx.doi.org/

10.1055/s-0034-1393036

Pneumologie 2015; 69: 608-615

(c) Georg Thieme Verlag KG

Stuttgart · New York

ISSN 0934-8387

Korrespondenzadresse Prof. Dr. med. Antje Prasse Abteilung für Pneumologie Medizinische Hochschule Hannover

Carl-Neuberg-Straße 1

30625 Hannover

Prasse.antje@mh-hannover.de

\section{Zusammenfassung}

Die idiopathische Lungenfibrose (IPF) ist die häufigste Form der Lungenfibrosen und tritt v.a. im höheren Lebensalter auf. Neben Alterungsprozessen und der genetischen Veranlagung ist ein wichtiger Risikofaktor das Zigarettenrauchen und die Exposition gegenüber lungentoxischen Substanzen. Der IPF liegt das Muster der Usual Interstitial Pneumonitis (UIP) histologisch zugrunde. Die Diagnosestellung ist komplex und sollte multidisziplinär im Ausschluss anderweitiger Erkrankungen erfolgen. Der einzige kurative Ansatz ist die Lungentransplantation. In den letzten Jahren hat es in der medikamentösen Therapie einen Durchbruch gegeben, erstmals wurden zwei Medikamente, Pirfenidon und Nintedanib, für die Behandlung der IPF zugelassen.

\section{Lernziele}

$\nabla$

In diesem Beitrag gewinnt der Leser einen Überblick über den Algorithmus zur Diagnosestellung der IPF und die aktuellen Therapieempfehlungen. Er soll dadurch in die Lage versetzt werden, die geeigneten Schritte zur Diagnosestellung zu veranlassen, u.U. eine medikamentöse Therapie einzuleiten und die Betreuung der behandelten Patienten zu gewährleisten.

\section{Einleitung \\ $\nabla$}

Die idiopathische Lungenfibrose oder auch idiopathische pulmonale Fibrose (IPF) ist eine in der Regel tödlich verlaufende Erkrankung, die mit einer zunehmenden Destruktion und Schrumpfung des Alveolargewebes einhergeht. Daten aus Großbritannien lassen eine Inzidenz von 8,6 Patienten/ 100000 Einwohner in Deutschland vermuten [1]. Die IPF gehört damit noch zu den seltenen Lungenerkrankungen und Medikamente für die IPF tragen einen „orphan drug“ Status [2]. Zeichen einer Lungenfibrose bzw. fibrotische Veränderungen im hochauflösenden (HR) CT-Thorax sind je-

\section{Abstract}

Idiopathic pulmonary fibrosis (IPF) is the most common idiopathic interstitial pneumonia and a disease of the elderly. Cigarette smoking and longterm exposure to substances harming alveolar epithelial cells are risk factors for the development of IPF. There is also evidence for a genetic susceptibility. IPF is defined as the idiopathic variant of Usual Interstitial Pneumonitis (UIP). Diagnosis of IPF is complex and based on the exclusion of other diseases associated with an UIP pattern. The only cure is lung transplantation. In the last years there was a breakthrough in the treatment of IPF. With pirfenidone and nintedanib there are now two compounds approved for the treatment of IPF.

doch ausgesprochen häufig in der Normalbevölkerung der über 50-Jährigen. [3] Eine Studie, die jüngst diese Altersgruppe in Framingham (USA) untersucht hat, kommt zu dem Schluss, dass fibrotische Veränderungen der Lunge bei $7 \%$ im HRCT festzustellen sind und dass $2 \%$ eine relevante Lungenfibrose aufweisen.

Wichtigste Risikofaktoren sind, neben dem Alter, männliches Geschlecht, das Zigarettenrauchen und die genetische Veranlagung (u. a. MUC5b Polymorphismus) [3-5].

\section{Definition}

Die idiopathische Lungenfibrose ist mit einem Anteil von 33\% die häufigste Form der Lungenfibrosen, die auch als interstitielle Pneumonien (IIP) klassifiziert werden. Aufgrund histologischer Merkmale erfolgt in der aktuellen Klassifikation eine Einteilung in 9 Subtypen [6]. Histologisch liegt der IPF die „Usual Interstitial Pneumonitis“ (UIP) zugrunde. Ein pathognomonisches Merkmal für die UIP sind die Fibroblasten - Foci, subepithe- 


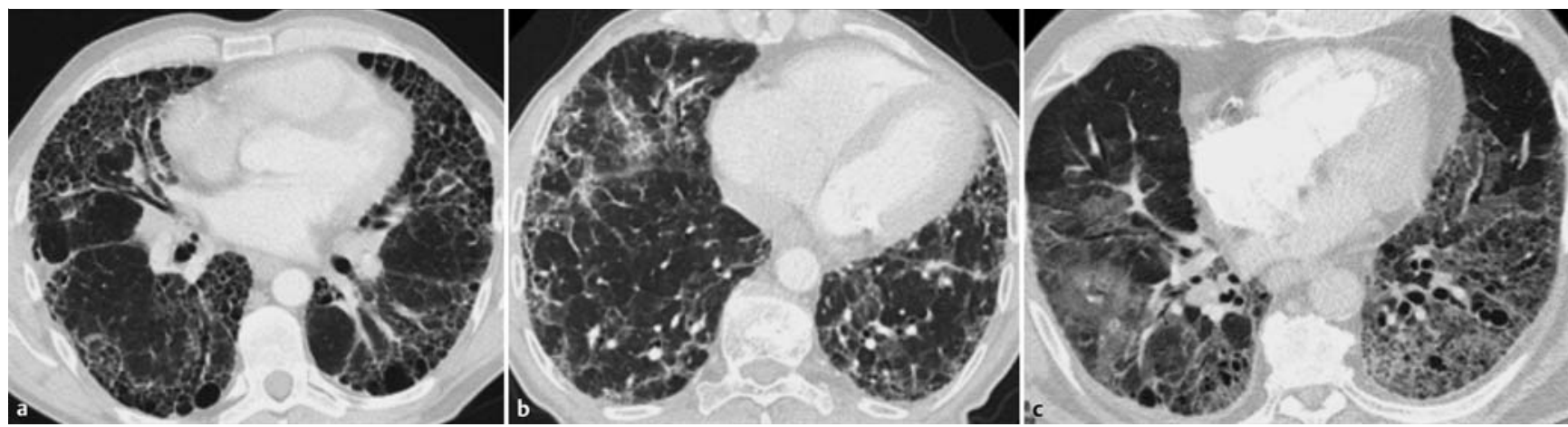

Abb. 1 UIP-Muster im HRCT-Thorax. In a Befund eines eindeutigen (definitive) UIP-Musters. In b ist eine mögliche (possible) UIP dargestellt, die histologisch einem UIP-Muster entsprach. In c ist ebenfalls eine mögliche (possible) UIP dargestellt, die histologisch einem UIP-Muster entsprach. Diese Patientin wurde trotz eines initial positiven ANCA-Befundes ausschließlich mit Pirfenidon anbehandelt. Fünf Monate später kam es zu einem pulmorenalen Syndrom (alveoläre Hämorraghie und rapid progressive Glomerulonephritis) im Rahmen einer mikroskopischen Polyangiitis. Das HRCT (c) entstand zu diesem Zeitpunkt.

liale Myofibroblastenansammlungen, die nur bei dieser Lungenfibroseform vorkommen. Darüber hinaus gilt als pathognomonisch der Nachweis einer zerstörten Lungenarchitektur, die regional heterogen ausgeprägt ist. Zudem kommt es häufig zur Neubildung von Honigwabenzysten, die aus bronchialem Ersatzgewebe aufgebaut sind.

Es müssen jedoch bei histologischem Nachweis einer UIP andere, ebenfalls zu einer UIP führende Erkrankungen ausgeschlossen werden, in erster Linie die Exogen-Allergische Alveolitis, eine Lungenbeteiligung bei rheumatologischen Systemerkrankungen und die Asbestose [5,7].

Männer erkranken deutlich häufiger als Frauen an einer IPF. Bei Frauen, die nie geraucht haben, ist eine IPF sehr selten. Aufgrund des Risikofaktors Zigarettenrauchen besteht bei nicht wenigen Patienten neben einer UIP auch ein Lungenemphysem. Gemäß dem aktuellen Konsensus der ATS und ERS wird die „emphysema-combined“ UIP nach Ausschluss anderer Ursachen der IPF subsumiert und nicht als eigenständiges Krankheitsbild angesehen [5].

\section{Klinik \\ $\nabla$}

Klinisch ist die IPF durch eine zunehmende Belastungsdyspnoe geprägt. Ein Reizhusten kann auftreten. Der Auskultationsbefund von Knisterrasseln beidseits basal ist ein Frühsymptom und häufig lange vor Veränderungen im Röntgen-Thorax nachweisbar. Das Krankheitsbild weist eine erhebliche Heterogenität auf. Neben lange Zeit stabilen und nur sehr langsam progredienten Verläufen gibt es immer wieder auch Patienten, die innerhalb der ersten 6 Monate nach Diagnosestellung versterben bzw. Krankheitsschübe mit akuter Verschlechterung innerhalb von 4 Wochen aufweisen. Letztere werden als akute Exazerbationen bezeichnet, und viele Patienten versterben im Rahmen einer akuten Exazerbation innerhalb weniger Tage. Bei ca. einem Drittel aller Patienten treten akute Exazerbationen auf. Im ersten Jahr nach Diagnosestellung sind ca. 10\% der Patienten betroffen.

$$
\begin{aligned}
& \text { Aufgrund der unterschiedlichen Verläufe ist die } \\
& \text { individuelle Prognose einzelner IPF-Patienten sehr } \\
& \text { schwierig abschätzbar. Treten akute Exazerbatio- } \\
& \text { nen auf, ist dies prognostisch ein sehr ungünstiges } \\
& \text { Zeichen }[5,8] \text {. }
\end{aligned}
$$

Darüber hinaus unterscheidet man die sporadisch auftretenden IPF-Formen von den familiären Formen, denen in der Regel eine genetische Mutation zugrunde liegt [9]. Im Vergleich zu den viel häufigeren sporadischen Fällen liegt das Erkrankungsalter der Patienten mit familiärer IPF deutlich niedriger, der Krankheitsverlauf ist häufig langsamer und spricht eher auf immunsuppressive Therapieversuche an [5].

Ein weiterer Subtyp ist die sporadische IPF mit Lungenemphysem, die sich ebenfalls klinisch von dem IPF-Subtyp ohne Lungenemphysem unterscheidet. Lungenfunktionell besteht bei diesen Patienten häufig eine viel geringere restriktive Ventilationsstörung. Gelegentlich weisen die Patienten mit dieser Unterform selbst im fortgeschrittenen Stadium noch eine normale Lungenfunktion auf. Bei diesen Patienten sind klinisch eine ausgeprägte Gasaustauschstörung und ein hoher Sauerstoffbedarf in Ruhe führend. Nicht selten kommt es deshalb erst verspätet zur Diagnosestellung. Zudem tritt bei diesem Subtyp häufig eine pulmonale Hypertonie auf $[2,10]$.

\section{Diagnosestellung}

\section{$\nabla$}

Die Diagnosestellung der IPF fußt auf einer multidisziplinären Zusammenschau der klinischen, laborchemischen, radiologischen und histologischen Befunde und sollte in einem Gremium von Experten aus der Pneumologie, Radiologie und Pathologie vorgenommen werden ( $\bullet$ Abb. 1 und - Abb. 2).

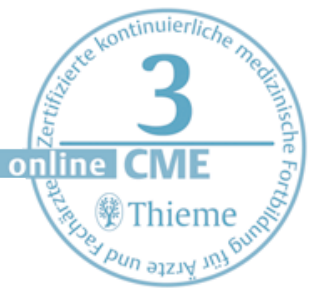




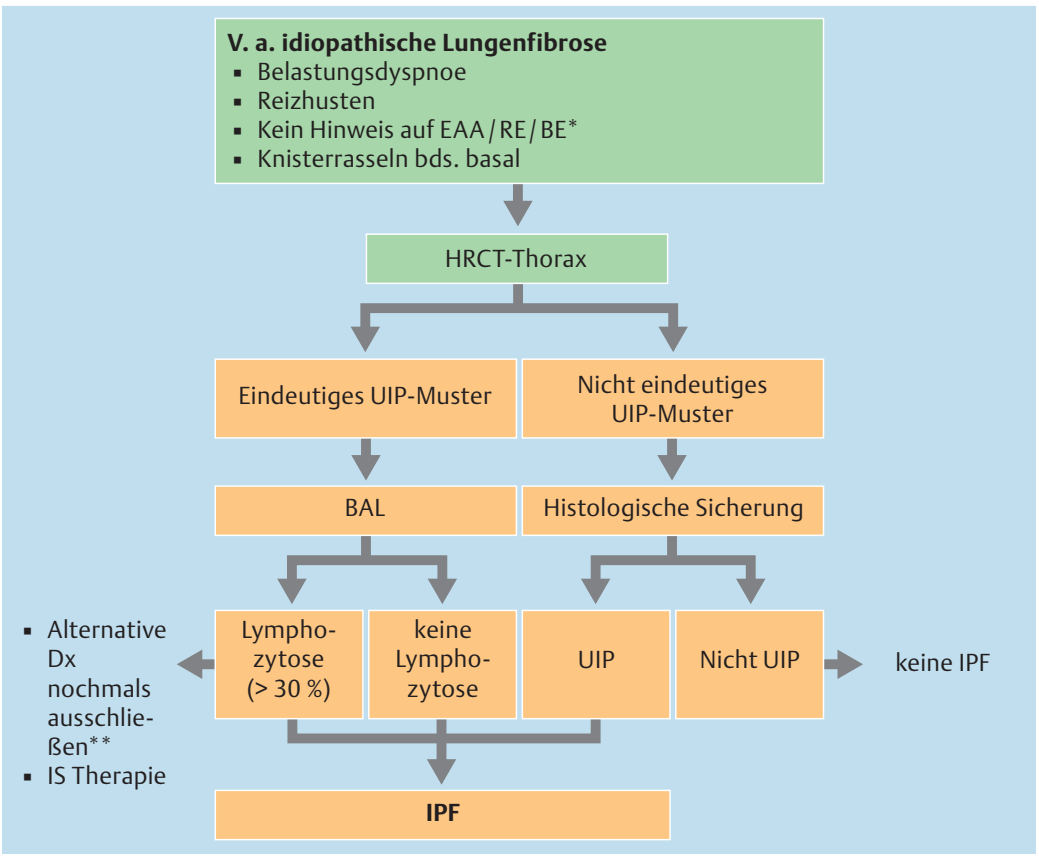

Abb.2 Algorithmus zur Diagnosestellung der IPF (modifiziert nach Raghu, G. et al [5]). Die Diagnosestellung sollte in einem multidisziplinär besetzten Gremium mit Vertretern der Fachrichtungen Pneumologie, Radiologie und Pathologie erfolgen. Wichtig ist insbesondere die exakte Abgrenzung gegenüber anderweitig entzündlich verursachten Erkrankungen/UIP-Formen, da bei diesen zumeist eine Indikation zur immunsuppressiven Therapie gegeben ist. * EAA: Exogenallergische Alveolitis; RE: Rheumatologische Erkrankung; BE: Berufserkrankung. ** mittels eingehender, gezielter Anamnese und laborchemisch.

Tab. 1 Graduierung des UIP-Musters im HRCT. UIP: Usual Interstitial Pneumonitis, HRCT: High Resolution Computer Tomography.

\begin{tabular}{lcc} 
Eindeutiges UIP-Muster & Mögliches UIP-Muster & Unvereinbar mit UIP \\
(4 Kriterien) & & \\
\hline - Honigwabenzysten & - Subpleurale und dorso- & - Oberfeld-Dominanz \\
und/oder Traktions- & basale Prädominanz & - Dominanz entlang der \\
bronchiektasien & - Retikuläre Verschat- & bronchovaskulären Bündel \\
- Subpleurale und dorso- & tungen & - Ausgedehnte Milchglas- \\
basale Prädominanz & - Fehlen von Läsionen, & verschattungen \\
- Retikuläre Verschat- & die unter „unverein- & - Multiple noduläre Verschat- \\
tungen & bar“ aufgelistet sind & tungen \\
- Fehlen von Läsionen, & & - Mosaikmuster/Air-trapping \\
die unter ,unverein- & & - Ausgedehnte Konsolidie- \\
bar“ aufgelistet sind & & rungen
\end{tabular}

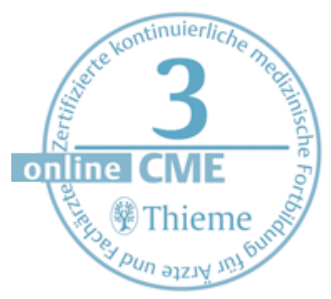

Eine passende Anamnese und Klinik sowie der Ausschluss anderweitiger Ursachen für eine Lungenfibrose (s.o.) bildet die Grundlage zur Diagnosestellung einer IPF. Nach den neuesten Empfehlungen der Fachgesellschaften wird der radiologische Befund gemäß der Wahrscheinlichkeit des Vorliegens einer UIP graduiert ( $\bullet$ Tab.1, - Abb.1). Liegt im HRCT-Thorax ein eindeutiges („definitive“) UIP-Muster vor, ist der histologische Nachweis einer UIP nicht erforderlich $[2,5,11]$. Bei möglichem („possible“) UIP-Muster im HRCT ist dieser jedoch erforderlich und kann über eine Keilresektion per Videothorakoskopie (VATS) oder bronchoskopisch per Kryobiopsie erfolgen. Auch bei eindeutigem UIP-Muster im HRCT führen die meisten europäischen Zentren eine BAL durch zum sicheren Ausschluss einer entzündlichen (durch T-Lymphozyten getriebenen) Erkrankung $[2,12]$.

Das derzeitige pathogenetische Konzept geht davon aus, dass ein repetitiver Schaden des Alveolarepithels Wundheilungsvorgänge auslöst, die dann zu fehlgeleiteten, abnormen Reparaturprozessen führen ( Abb.3) $[9,13]$.

Vermutlich gibt es nicht nur einen uniformen Schädigungsreiz (Noxe), sondern eine Vielzahl an Faktoren, die zur Schädigung des Alveolarepithels führen. Genetische Mutationen, die den familiären IPF-Formen zugrunde liegen, lösen häufig eine Schädigung des Alveolarepithels aus. So können Surfactant-Protein-C-Mutationen zu einer Akkumulation des Proteins in der Alveolarepithel-Zelle führen und dadurch ein vermehrtes $\mathrm{Ab}$ sterben induzieren [14]. Insbesondere die Schädigung der Stammzelle des Alveolarepithels (Alveolarepithel Zelle Typ II) durch Altersvorgänge, zellschädigende Noxen (Zigarettenrauchen, ...) führt wohl dazu, dass normales Alveolarepithel nach Schädigung nur unzureichend nachgebildet werden kann [15]. Die persistierende Schädigung (Wunde) des Alveolarepithels triggert Wundheilungsvorgänge und führt zum permanenten Einstrom diverser Vorläuferzellen und vor allem von Fibroblasten. Diese versuchen, mit Ersatzgewebe die Wunde zu schließen. Hierbei kommt es zu einer erheblichen Zellproliferation und zur Produktion von extrazellulärer Matrix (Bindegewebe). Beide Prozesse destruieren zunehmend den Alveolarraum, sodass zuletzt kein Alveolargewebe mehr vorhanden ist.

\section{Medikamentöse Therapie \\ $\nabla$}

Das Jahr 2014 hat einen Paradigmenwechsel in der Behandlung der IPF eingeleitet [16].

Lange Zeit galt die IPF als medikamentös nicht modulierbar, doch die Ergebnisse zweier großer multizentrischer, Plazebo-kontrollierter Phase III Studien belegen eindeutig, dass der mittlere Verlust an FVC (forcierte Vitalkapazität) im Jahresverlauf bei den mit Verum behandelten Patienten signifikant abgemildert wird.

Obwohl dies als ein Durchbruch in der IPF-Therapie zu werten ist, ist die Erkrankung jedoch nach wie vor nicht heilbar. Lungenfunktionsverbesserungen sind nur bei einem kleinen Teil der Patienten unter medikamentöser Therapie zu erwarten und erreichen nicht die Dimension einer restitutio ad integrum. 


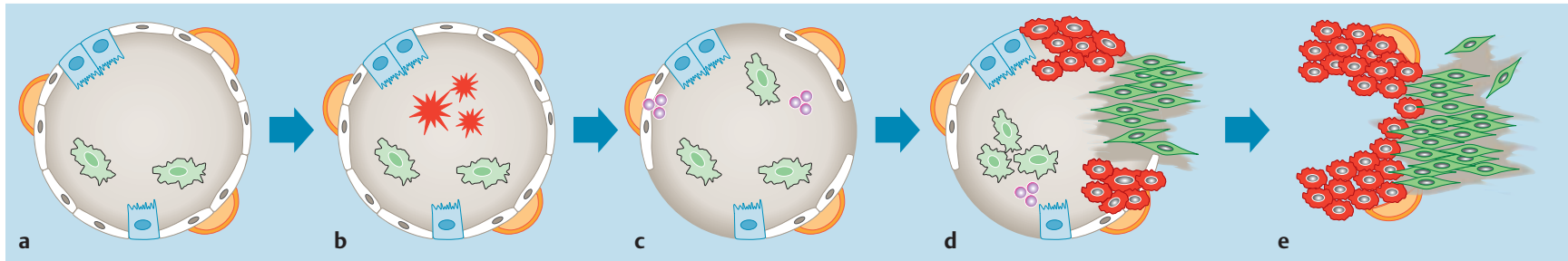

Abb. 3 Pathogenese der Idiopathischen Lungenfibrose. In a ist eine normale Alveole dargestellt, die von Alveolarepithelzellen ausgekleidet ist und von einem Netz aus Kapillaren umgeben wird. Die Alveolarepithelzelle Typ II ist die Stammzelle des Alveolarepithels. Es gibt viele Hinweise, dass diese AEC-II-Zellen bei der IPF geschädigt sind und nicht mehr die Regeneration des Alveolarepithels bewerkstelligen. Hier spielen genetische Veranlagung, Alterungsprozesse und eine Schädigung durch toxische Substanzen eine wichtige Rolle. Vermutlich kommt es bei IPF-Patienten zu rezidivierenden Schäden am Epithel, und es entstehen Wunden (b, c). Wundheilungsprozesse setzen ein, Entzündungszellen und Vorläuferzellen strömen ein (d). Da die Wunde nicht durch die geschädigte Stammzelle des Alveolarepithels geschlossen werden kann, versuchen anstatt dessen Fibroblasten und bronchiale Epithelzellen diese zu reparieren. Es bildet sich überschießendes Ersatzgewebe aus, welches die normale Alveolarstruktur zerstört (e).

Der einzige kurative Therapieansatz ist nach wie vor die Lungentransplantation [2,5]. Aufgrund des Risikos für die oben geschilderten akuten Exazerbationen sollte bei jüngeren Patienten bereits bei Erstdiagnose die Anbindung an ein Lungentransplantations-Zentrum erfolgen, sofern keine Kontraindikation zur Lungentransplantation besteht.

Weiterhin sollte regelmäßig getestet werden, inwieweit eine supplementäre Versorgung mit Sauerstoff indiziert ist. Sehr früh im Krankheitsverlauf kann bereits ein erheblicher Abfall des Sauerstoffpartialdruckes bei geringer körperlicher Belastung, z.B. im Rahmen eines 6-Minuten-Gehtestes, auftreten. Fällt bei einem solchen Test die Sättigung unter $85 \%$ bzw. der Sauerstoffpartialdruck unter $50 \mathrm{mmHg}$, ist die Indikation zur Versorgung mit einem mobilen Sauerstoffgerät gegeben. In Ruhe ergibt sich eine Indikation bei einem $\mathrm{PaO}_{2}<55 \mathrm{mmHg}$. Außerdem finden sich zunehmend Hinweise, dass rehabilitative Maßnahmen den Krankheitsverlauf möglicherweise günstig beeinflussen. Für viele Patienten und ihre Angehörigen ist das frühzeitige Angebot von palliativmedizinischen Maßnahmen hilfreich.

\section{Medikamentöse Therapie \\ $\nabla$}

Die europäische IFIGENIA-Studie hatte 2006 einen verminderten FVC-Verlust unter der TripelTherapie N-Acetylcystein + Azathioprin+Prednison versus Prednison+Azathioprin ergeben [17] Diese Studie wurde in der Folge insbesondere in den USA erheblich angegriffen, da sie keinen eigentlichen Plazeboarm umfasste. Vom NIH wurde deshalb eine Studie (PANTHER) aufgelegt, die diese Lücke schließen sollte. Aufgrund von vermehrten Hospitalisierungen und Todesfällen musste der Tripel-Therapiearm vor Beendigung der Studie abgebrochen werden, und es kam zu einem entsprechenden Warnhinweis für die Tripel-Therapie [18]. Auf der Basis dieser Daten spricht sich die aktuelle Leitlinie „negativ“ gegen eine TripelTherapie bei IPF-Patienten aus [2]. Nur in Ausnahmefällen, bei klarem Therapieansprechen bzw. beim Nachweis einer Lymphozytose oder von Autoimmunprozessen kann diese als Dauertherapie sinnvoll sein. Vor kurzem wurde die Analyse des Therapiearmes mit Acetylcystein $1800 \mathrm{mg} / \mathrm{d}$ mono versus Plazebo publiziert. Weder im primären Endpunkt noch in einem der sekundären Endpunkte war ein signifikantes Ergebnis festzustellen, sodass diese Studie zusammengefasst keinen Benefit der N-Acetylcystein Monotherapie belegt [19]. Jüngste Ergebnisse, die auf dem ATS 2015 berichtet wurden, zeigen, dass die unterschiedlichen Behandlungsarme in der PANTHERStudie möglicherweise einem genetischen Bias unterlagen. Das genetische Risikoprofil der Patienten in den einzelnen Behandlungsarmen war signifikant unterschiedlich. Möglicherweise gibt es zudem eine Assoziation mit bestimmten Polymorphismen im TOLLIP-Gen, die mit einem guten bzw. mit einem unvorteilhaften Therapieansprechen auf NAC einhergehen sollen.

Eine weitere Studie hat retrospektiv die Daten mehrerer Plazebogruppen von NIH-Studien zur IPF, die negativ verlaufen sind, im Hinblick auf die Einnahme von Antacida untersucht [20]. Die Daten zur Medikamenteneinnahme und Anamnese bzgl. einer Refluxsymtomatik wurden in den jeweiligen Studien zwar prospektiv erfasst, wurden jedoch erst nach Abschluss der die anderen Medikamente betreffenden Studien retrospektiv ausgewertet. Interessanterweise zeigt diese Studie, dass es bei den Patienten, die Protonenpumpeninhibitoren oder H2-Blocker einnahmen, zu einem verminderten Auftreten von akuten Exazerbationen und Hospitalisationen kam. Die Studie war nicht ausreichend groß, um zu klären, ob auch Patienten, die keine Refluxsymptomatik aufweisen, von einer Anti-Refluxtherapie profitieren. In diesem Zusammenhang ist jedoch darauf hinzuweisen, dass die Inzidenz an Reflux und Mikroaspirationen bei IPF-Patienten deutlich erhöht ist. Unter anderem scheinen die nicht unerheblichen Distorsionen des Mediastinums und insbesondere des Tracheobronchialbaumes bei voranschreitender Schrumpfung des Lungengewebes das Auftreten von Reflux und Mikroaspiration zu begünstigen.

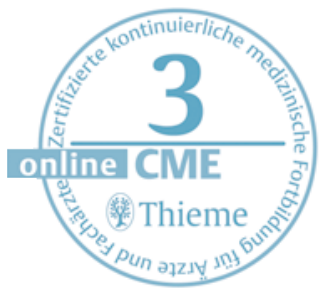


Auf der Basis dieser Daten wird der Einsatz von Antacida in den aktuellen Leitlinien "bedingt“ empfohlen [21].

Auf Grundlage der CAPACITY-Studien wurde Pirfenidon in Europa bereits seit Herbst 2011 zur Behandlung der IPF mit milder bis moderater Lungenfunktionseinschränkung zugelassen [22].

Die FDA forderte vor der Zulassung noch eine weitere Phase-III-Studie, sodass in den USA die Zulassung erst nach Publikation der ASCEND-Studie im Herbst 2014 erfolgte [23]. Der Wirkmechanismus von Pirfenidon ist bisher nur unzureichend verstanden. Das Molekül scheint jedoch in mehrere zelluläre Signalwege einzugreifen, u.a. wird die TGF-beta-Produktion vermindert. In die ASCENDStudie wurden 555 Patienten eingeschlossen, die im HRCT entweder ein eindeutiges UIP-Muster oder ein wahrscheinliches UIP-Muster, dann jedoch zusätzlich mit histologischer Sicherung, haben mussten. Patienten, die neben ihrer idiopathischen Lungenfibrose noch ein Emphysem aufwiesen, waren ausgeschlossen. Mit einer minimalen FVC von $\geq 50 \%$ und einer Diffusionskapazität von $\geq 30 \%$ wurden lungenfunktionell leicht bis moderat eingeschränkte IPF-Patienten untersucht. Der primäre Endpunkt war der Verlust an FVC im Verlauf über 1 Jahr (52 Monate) und dieser wurde erreicht. Im Vergleich zur Plazebogruppe fiel bei den mit Pirfenidon behandelten Patienten die FVC im Mittel um $116 \mathrm{ml}$ weniger ab. Auch im 6-Minuten-Gehtest fand sich eine signifikant geringere Abnahme der Gehstrecke unter Pirfenidon. Die gepoolte Analyse der Pirfenidon-Studien (ASCEND und CAPACITY I+ II) ergab einen Überlebensvorteil der Pirfenidon behandelten Patienten über 52 Wochen. Bei einer allerdings insgesamt sehr geringen Anzahl von Todesfällen in beiden Studien verstarben 3,5\% der mit Pirfenidon behandelten Patienten gegenüber 6,7\% in der Plazebogruppe, entsprechend einer relativen Sterberisikoreduktion von 0,7. Ob diese gepoolte Analyse jedoch bei unterschiedlichen Ein- und Ausschlusskriterien der Studien zu Pirfenidon überhaupt möglich ist, ist umstritten und wird beispielsweise von der FDA abgelehnt [21,23]. Die Erfahrung zahlreicher Anwendungsbeobachtungsstudien ergab darüber hinaus, dass Pirfenidon bei progredientem Lungenfunktionsverlust die Erkrankung stabilisieren kann [24]. Pirfenidon ist in Tabletten mit $267 \mathrm{mg}$ erhältlich und sollte über 2 Wochen auf eine Dosierung von 3 mal 3 Tabletten $(2403 \mathrm{mg} / \mathrm{d})$ gesteigert werden. Nebenwirkungen unter Pirfenidon-Therapie sind nicht selten. Vor allem gastrointestinale Nebenwirkungen wie Übelkeit, Appetitlosigkeit, Erbrechen, Sodbrennen und Diarrhoen können auftreten. Eine symptomatische Therapie mit Protonenpumpeninhibitoren oder Loperamid kann hilfreich sein. Gelegentlich kann jedoch eine resultie- rende Gewichtsabnahme die Beendigung der Therapie notwendig machen. Zudem können Hautausschläge auftreten und die Photosensibilität wird erheblich gesteigert. Patienten, die das Medikament einnehmen, sollten angehalten werden, Sonnenlichtexposition zu meiden und ggf. Protektionsmaßnahmen zu ergreifen, wie z.B. Sonnencreme mit dem Lichtschutzfaktor 50 aufzutragen. Selten können Müdigkeit und psychiatrische Nebenwirkungen (Depression/Psychose) auftreten. Bei letzteren sind insbesondere die Medikamenteninteraktionen problematisch, so vor allem die gleichzeitige Einnahme von Fluvoxamin (und anderen Serotinin-Wiederaufnahmehemmern), da beide Medikamente über den CYP1A2 verstoffwechselt werden. Zudem ist bei der Einnahme von Ciprofloxacin, Amiodaron und Propafenon Vorsicht geboten. Pirfenidon wird über die Nieren ausgeschieden, und es besteht eine Kontraindikation bei schwerer Niereninsuffizienz (GFR <30) bzw. Dialysepflichtigkeit. Engmaschig sollten Gamma-GT, Transaminasen und Bilirubin bei Patienten mit leicht- bis mittelgradig eingeschränkter Leberfunktion kontrolliert werden. Grapefruitsaft sollte gemieden werden.

Nintedanib, ein Multi-Tyrosinkinase-Inhibitor wurde für die Behandlung der idiopathischen Lungenfibrose durch die EMEA zugelassen, und die Verordnung für die Indikation IPF ist seit April 2015 in Deutschland möglich $[27,28]$.

Interessanterweise wurde das Medikament ursprünglich für die Onkologie entwickelt und wird seit Januar 2015 bereits zur Therapie des nichtkleinzelligen Bronchialkarzinoms eingesetzt. Auch für andere Karzinomarten wird die Substanz derzeit getestet. Nintedanib inhibiert unter anderem die Tyrosinkinasen VEGF, PDGF und FGF und wurde ursprünglich zur Antiangiogenese entwickelt [29]. Es hemmt jedoch nicht nur die Proliferation von Endothelzellen, sondern auch die Proliferation von Fibroblasten. Die vierarmige Phase-II-Studie (TOMORROW) ergab, dass Patienten in dem Arm mit der höchsten Dosis Nintedanib (2 mal $150 \mathrm{mg} / \mathrm{d}$ ) einen signifikant geringeren Lungenfunktionsverlust aufwiesen als Patienten unter Plazebo. Dieses Signal konnte in der zweiarmigen Phase-III-Studie (INPULSIS) belegt werden $[27,28]$. In beiden INPULSIS-Studien I und II war der FVC-Abfall bei den mit Nintedanib behandelten Patienten im Mittel signifikant niedriger als unter Plazebo. Auch in dieser Studie wurde der Verlust an FVC über ein Jahr erfasst und ergab eine Differenz zu Plazebo von $111 \mathrm{ml}$. Auch wenn die Einschlusskriterien und die Studiendauer der INPULSIS-Studie nicht exakt der ASCEND-Studie entsprachen, so erscheint die Größenordnung des FVC-Gewinnes unter Nintedanib versus Pirfenidon im Vergleich zu den jeweiligen Plazebogruppen in etwa gleich hoch zu sein. Die TOMORROW-Studien hatten darüber hinaus einen ersten 
Hinweis darauf ergeben, dass unter Nintedanib das Risiko für das Auftreten von akuten Exazerbationen gesenkt werden kann. Die INPULSIS-Studien I und II konnten dies jedoch nur teilweise bestätigen. Insgesamt war die Anzahl an akuten Exazerbationen in den beiden Studienarmen unerklärlicherweise unterschiedlich hoch. Der INPULSIS-II-Studienarm, in dem mehr akute Exazerbationen auftraten, zeigte einen signifikanten Einfluss von Nintedanib auf das Auftreten akuter Exazerbationen. Auch die gepoolte Analyse des Auftretens an akuten Exazerbationen, die durch ein Expertengremium bestätigt worden waren, ergab, dass Nintedanib das Risiko hierfür senkt [27]. Zudem zeigt die gepoolte Analyse der TOMORROW- und INPULSIS-Studien eine relative Sterberisikoreduktion von 0,7 unter Nintedanibtherapie, vergleichbar mit dem Effekt von Pirfenidon [21]. Auch für Nintedanib ist jedoch umstritten, ob die gepoolte Analyse mehrerer Studien statistisch korrekt ist. Die genannten Studien belegen zudem, dass auch unter Nintedanib häufig Nebenwirkungen auftreten. Führend waren gastrointestinale Nebenwirkungen, wie Diarrhoen, Sodbrennen, Übelkeit, Erbrechen, Appetitlosigkeit und eine Gewichtsabnahme. Da es sich um ein Präparat handelt, welches durch seine Antiangiogenese möglicherweise die Wundheilung beeinflusst, wird derzeit empfohlen, das Präparat vor geplanten Operationen abzusetzen.

\section{Fazit}

\section{$\nabla$}

Auf Basis mehrerer Studien, die im Jahr 2014 publiziert wurden, kam es zu einem Paradigmenwechsel bzgl. der Behandlung der IPF. Die IPF gilt seitdem als medikamentös behandelbar. Mit Nintedanib und Pirfenidon stehen nun zwei für die IPF zugelassene Medikamente zur Verfügung, die in der aktuellen ATS/ERS Leitlinie [21] neben dem Einsatz von Antacida „bedingt“ zur Therapie empfohlen werden.

Die Zulassung medikamentöser Therapien für die IPF war ein wichtiger erster Schritt. Die zahlreichen Medikamente, die sich derzeit in der klinischen Testung befinden, sowie das wieder entfachte Interesse der Pharmaindustrie an diesem Krankheitsbild lassen hoffen, dass dies erst der Anfang einer langen Entwicklung ist. Unklar ist auch, inwieweit eine Kombinationstherapie von Nintedanib und Pirfenidon möglich und erfolgversprechend ist. Aktuell gibt es noch keinen medikamentösen Therapieansatz mit kurativer Wirkung. Der immense Erkenntnisgewinn, der aus den neu zur Verfügung stehenden Therapiestrategien in den nächsten Jahren erwachsen wird, wird jedoch sicher dazu beitragen, dass wir uns diesem Ziel nähern. Vordringlich für die nächsten Jahre wird es zudem sein, parallel zur Produkt- entwicklung, Konzepte zu entwickeln, die helfen, das individuelle Therapieansprechen vorherzusagen.

Darüber hinaus gilt es nun, Patienten mit IPF frühzeitig zu identifizieren und eine exakte Diagnosestellung anzustreben. Wichtig ist insbesondere, die entzündlichen Formen der UIP von der IPF klar abzugrenzen, da in den aktuellen Therapieempfehlungen die immunsuppressive Therapie nicht mehr empfohlen wird.

\section{Interessenkonflikt}

$\nabla$

Boehringer Ingelheim Pharma: Referententätigkeit und Beratung; Roche: Referententätigkeit und Beratung; Biogen Idec: wissenschaftliches Projekt.

\section{Literatur}

1 Hutchinson J, Fogarty A, Hubbard R et al. Global incidence and mortality of idiopathic pulmonary fibrosis: a systematic review. Eur Respir J 2015; 46: 795-806

2 Behr ], Gunther A, Ammenwerth W et al. [German guideline for diagnosis and management of idiopathic pulmonary fibrosis]. Pneumologie 2013; 67: 81-111

3 Hunninghake GM, Hatabu H, Okajima Y et al. MUC5B promoter polymorphism and interstitial lung abnormalities. N Engl J Med 2013; 368: 2192-2200

4 Seibold MA, Wise AL, Speer MC et al. A common MUC5B promoter polymorphism and pulmonary fibrosis. $\mathrm{N}$ Engl ] Med 2011; 364: 1503-1512

5 Raghu G, Collard HR, Egan JJ et al. An official ATS/ERS/JRS/ ALAT statement: idiopathic pulmonary fibrosis: evidence-based guidelines for diagnosis and management. Am J Respir Crit Care Med 2011; 183: 788 - 824

6 Travis WD, Costabel U, Hansell DM et al. An official American Thoracic Society/European Respiratory Society statement: Update of the international multidisciplinary classification of the idiopathic interstitial pneumonias. Am J Respir Crit Care Med 2013; 188: 733 -748

7 Morell F, Villar A, Montero MA et al. Chronic hypersensitivity pneumonitis in patients diagnosed with idiopathic pulmonary fibrosis: a prospective case-cohort study. Lancet Respir Med 2013; 1: 685-694

8 Collard HR, King TElr. Idiopathic pulmonary fibrosis and nonspecific interstitial pneumonia should stay separate. Eur Respir J 2008; 31: 1141 - 1142; author reply 2-3

9 King TEJr, Pardo A, Selman M. Idiopathic pulmonary fibrosis. Lancet 2011; 378: 1949-1961

10 Cottin V, Nunes H, Brillet PY et al. Combined pulmonary fibrosis and emphysema: a distinct underrecognised entity. Eur Respir J 2005; 26: 586-593

11 American Thoracic Society. Idiopathic pulmonary fibrosis: diagnosis and treatment. International consensus statement. American Thoracic Society (ATS), and the European Respiratory Society (ERS). Am J Respir Crit Care Med 2000; 161: 646-664

12 Ohshimo S, Bonella F, Cui A et al. Significance of bronchoalveolar lavage for the diagnosis of idiopathic pulmonary fibrosis. Am J Respir Crit Care Med 2009; 179: $1043-$ 1047

13 Selman M, King TE, Pardo A. Idiopathic pulmonary fibrosis: prevailing and evolving hypotheses about its pathogenesis and implications for therapy. Ann Intern Med 2001; 134: 136-151

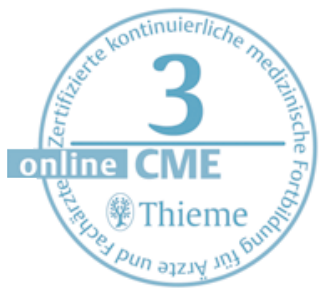


14 Korfei M, Ruppert C, Mahavadi P et al. Epithelial endoplasmic reticulum stress and apoptosis in sporadic idiopathic pulmonary fibrosis. Am J Respir Crit Care Med 2008; 178: $838-846$

15 Selman M, Pardo A. Revealing the pathogenic and agingrelated mechanisms of the enigmatic idiopathic pulmonary fibrosis. an integral model. Am J Respir Crit Care Med 2014; 189: 1161 - 1172

16 Hunninghake GM. A new hope for idiopathic pulmonary fibrosis. N Engl J Med 2014; 370: $2142-2143$

17 Demedts M, Behr J, Buhl R et al. High-dose acetylcysteine in idiopathic pulmonary fibrosis. N Engl J Med 2005; 353: $2229-2242$

18 Raghu G, Anstrom KJ, King TEJr et al. Prednisone, azathioprine, and $\mathrm{N}$-acetylcysteine for pulmonary fibrosis. $\mathrm{N}$ Engl J Med 2012; 366: 1968 - 1977

19 Martinez FJ, de Andrade JA, Anstrom KJ et al. Randomized trial of acetylcysteine in idiopathic pulmonary fibrosis. $\mathrm{N}$ Engl J Med 2014; 370: 2093 - 2101

20 Lee JS, Collard HR, Anstrom KJ et al. Anti-acid treatment and disease progression in idiopathic pulmonary fibrosis: an analysis of data from three randomised controlled trials. Lancet Respir Med 2013; 1: 369 - 376

21 Raghu G, Rochwerg B, Zhang Y et al. An Official ATS/ERS/ IRS/ALAT Clinical Practice Guideline: Treatment of Idiopathic Pulmonary Fibrosis. An Update of the 2011 Clinical Practice Guideline. Am J Respir Crit Care Med 2015; 192: e3-e19
22 Noble PW, Albera C, Bradford WZ et al. Pirfenidone in patients with idiopathic pulmonary fibrosis (CAPACITY): two randomised trials. Lancet 2011; 377: 1760 - 1769

23 King TEJr, Bradford WZ, Castro-Bernardini S et al. A phase 3 trial of pirfenidone in patients with idiopathic pulmonary fibrosis. N Engl J Med 2014; 370: 2083 -2092

24 Loeh B, Drakopanagiotakis F, Bandelli GP et al. Intraindividual response to treatment with pirfenidone in idiopathic pulmonary fibrosis. Am J Respir Crit Care Med 2015; 191: $110-113$

25 Harari S, Caminati A, Albera C et al. Efficacy of pirfenidone for idiopathic pulmonary fibrosis: An Italian real life study. Respir Med 2015; 109: 904 - 913

26 Bonella F, Wessendorf TE, Costabel U. [Clinical experience with pirfenidone for the treatment of idiopathic pulmonary fibrosis]. Dtsch Med Wochenschr 2013; 138: 518 523

27 Richeldi L, du Bois RM, Raghu G et al. Efficacy and safety of nintedanib in idiopathic pulmonary fibrosis. N Engl J Med 2014; 370: 2071 - 2082

28 Richeldi $L$, Costabel $U$, Selman $M$ et al. Efficacy of a tyrosine kinase inhibitor in idiopathic pulmonary fibrosis. N Engl J Med 2011; 365: 1079-1087

29 Wollin L, Wex E, Pautsch A et al. Mode of action of nintedanib in the treatment of idiopathic pulmonary fibrosis. Eur Respir J 2015; 45: 1434 - 1445

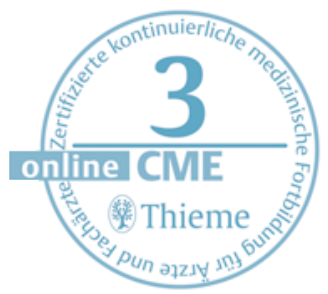




\section{CME-Fragen Die idiopathische Lungenfibrose}

1 Welche Läsion ist typisch für ein UIP-Muster im HRCT ?

A Milchglasverschattung

B Fleckschatten

C subpleurale Dominanz der Läsionen

D Lymphadenopathie

E Kavernen

2 Der typische Auskultationsbefund der IPF ist

A ein Brummen.

B v.a. in den Oberfeldern zu hören.

C ein exspiratorisches Rasseln.

D blande.

E sehr frühzeitig zu hören.

\section{Welche Aussage zur IPF trifft zu?}

3 A Genetische Risikofaktoren sind nicht bekannt. B Die IPF ist eine Autoimmunerkrankung.

C Zigarettenrauchen ist ein Risikofaktor.

D Tritt häufiger bei Frauen auf.

E Familiäre Formen wurden nicht beschrieben.

4 Bei Patienten mit möglichem (possible) UIP-Muster im HRCT

A ist eine Indikation zur Kortikosteroidbehandlung gegeben.

B ist eine Lungenbiopsie lt. Leitlinie zur Diagnosestellung IPF erforderlich.

C reicht zur Diagnosestellung eine BAL mit dem Ausschluss einer Lymphozytose aus.

D liegt eine IPF vor.

E sollte immer eine orale Antikoagulation erfolgen.

\section{Welche Aussage zur Therapie der IPF trifft zu?}

A Eine Phase-III-Studie zur oralen Antikoagulation war positiv.

B In zwei klinischen NIH-Studien zu Sildenafil wurde der primäre Endpunkt erreicht.

c Die immunsuppressive Triple-Kombination Prednison $+A Z A+N A C$ wird in den aktuellen Leitlinien bedingt empfohlen.

D In einer kombinierten Analyse dreier NIH-Studien verringert der Einsatz von Antazida das Risiko für akute Exazerbationen.

E Die Monotherapie mit N-Acetylcystein wird von der aktuellen Leitlinie als stark positiv (Evidenzlage 1A) bewertet.
6 Welche Aussage zur Therapie mit Pirfenidon trifft nicht zu?

A Die häufigste Nebenwirkung sind gastrointestinale Beschwerden.

B Patienten sollen bei Sonnenlichtexposition Lichtschutzfaktor 50 auftragen.

C Die Leberenzyme sollten unter der Therapie mit Pirfenidon kontrolliert werden.

D Pirfenidon sollte nicht mit Protonenpumpeninhibitoren kombiniert werden.

E Selten kann es unter dem Medikament zu Psychosen kommen.

7 Welche Aussage zur Therapie mit Nintedanib trifft nicht zu?

A Die häufigste Nebenwirkung sind gastrointestinale Beschwerden.

B Die Leberenzyme sollten unter der Therapie mit Nintedanib kontrolliert werden.

C Männer sollten keine Kinder zeugen, wenn sie Nintedanib einnehmen.

D Nintedanib ist auch für die Therapie des Bronchialkarzinoms zugelassen.

E Vor elektiven Operationen sollte das Medikament abgesetzt werden.

8 Welche Aussage zur akuten Exazerbation der IPF trifft zu?

A Die Mortalität der akuten Exazerbation ist sehr hoch.

B Die maschinelle Beatmung hat i.R. einen günstigen Einfluss auf den Krankheitsverlauf.

C Es liegen dieselben Pathomechanismen wie bei der Exazerbation der COPD zugrunde.

D Ca. die Hälfte aller IPF-Patienten erleidet im ersten Jahr nach Diagnosestellung eine Exazerbation.

E Klassischerweise finden sich im HRCT-Thorax neuaufgetretene retikuläre Veränderungen.

9 Welcher Satz ist falsch?

A Die IPF ist eine Erkrankung des alten Menschen.

B Das eindeutige (definitive) UIP-Muster tritt nur bei der IPF auf.

C Im HRCT hat die IPF eine dorsobasale Dominanz.

D Die familiären IPF-Formen manifestieren sich früher als die sporadischen.

E Emphysema combined UIP-Formen werden der IPF subsumiert.

Welche Erkrankung kann kein UIP-Muster hervorrufen?

A Exogene Allergische Alveolitis

B Asbestose

C Sarkoidose

D Lungenemphysem

E Rheumatoide Arthritis 